\title{
HOCHWERTIGE SPEZIALSEMINARE FÜR FORTGESCHRITTENE
}

\section{Sehr geehrte Frau Kollegin, sehr geehrter Herr Kollege!}

Nachdem wir immer mehr in der TCM und Aurikulomedizin erfahrene KollegInnen betreuen, wird uns immer wichtiger, auch für weit Fortgeschrittene sinnvolle Weiterbildung zu veranstalten. $Z u$ diesen Kursen, die auf unseren Seiten „OGKA-aktuell“ in dieser Zeitschrift angekündigt werden, lade ich Sie sehr herzlich ein, denn nichts ist wichtiger als laufend sein Wissen aufzufrischen. Auch mir persönlich ist immer noch jede Veranstaltung nicht nur eine Quelle für neues Wissen, sondern vor allem auch ein Turbo für meine eigene Motivation und Freude an der Arbeit. Bitte nutzen Sie also dieses Angebot und informieren Sie sich bei uns telefonisch, welches Angebot für Sie persönlich von höchstem Nutzen wäre. In diesen Zusammenhang sind unser Jahressymposium und der TAO-Kongress unsere Leitveranstaltungen.

Beim Jahressymposium in Bad Hall (Nähe Linz) vom 30.5.-1.6.2019 hat Dr. Reininger wieder ein unglaublich buntes Spektrum von Themen vorbereitet:Wie Sie dem OGKA-aktuell dieser Zeitschrift entnehmen können, reichen die Vorträge von „Biophotonen“ über neueste Erkenntnisse aus der Aurikulomedizin bis zu „TCM-Klassikern“. Im Rahmen dieses Symposiums werden wir mit einer tollen Festveranstaltung unser 25-jähriges Bestehen feiern. Zudem wird meine Nachfolgerin gewählt und damit eine neue Ära der OGKA eingeleitet - Also gleich eine Vielzahl von Gründen, bei unserem Jahressymposium dabei zu sein!

Beim heurigen, internationalen ,TAO-Kongress“ in Graz widmen wir uns vom 26.-28.9.2019 dem hochaktuellen Themenkreis ,TCM für Fertilität und Kinderwunsch" ". Es ist uns gelungen, führende internationale Spezialisten dieses Fachgebiets zu engagieren. Insgesamt werden bei unserem Kongress 45 ReferentInnen unterrichten. Zudem hat Frau Kollegin Olivia KrammerPojer die Möglichkeit geschaffen, dass unsere TeilnehmerInnen im Rahmen unserer Kurse DAS internationale Standard-Diplom für „Fertilität und Kinderwunsch“ erlangen können. Bei Interesse ersuche ich Sie, Detailinformationen in unserem Büro abzufragen.

Ich möchte Ihr Augenmerk noch auf unsere TAOVor- und -Nachkurse lenken. Die Idee war: Wenn wir schon so viele hochinteressante Referenten bei uns in Graz haben, dann wollen wir sie nicht nur kurz hören, sondern mit ihnen intensive Seminare anbieten, um vom Wissen dieser Spezialisten noch mehr profitieren zu können.

So würde ich mich sehr freuen, Sie beim Jahressymposium in Bad Hall oder beim TAO-Kongress in Graz begrüßen zu dürfen!

Ihr

Prof. Dr. med. Leopold Dorfer

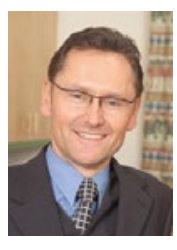

Prof. Dr. med. Leopold Dorfer

Präsident der Österreichischen Gesellschaft für Kontrollierte Akupunktur und TCM (OGKA)

Glacisstraße 7, A-8010 Graz

Tel. $+43316 / 374050$

E-Mail:office@ogka.at, Internet:www.ogka.at 